\title{
RSSI-Based Forwarding for Multihop Wireless Sensor Networks
}

\author{
Azlan Awang, Xavier Lagrange, and David Ros \\ Institut TELECOM / TELECOM Bretagne, \\ Networks, Security and Multimedia dept. \\ 2 rue de la Châtaigneraie, CS 17607, \\ 35576 Cesson Sévigné Cedex \\ Université européenne de Bretagne, France \\ \{azlan.awang, xavier. lagrange, david.ros\}@telecom-bretagne.eu \\ http://www.telecom-bretagne.eu
}

\begin{abstract}
In a multihop Wireless Sensor Network (WSN), a salient point among routing protocols that do not depend on network topology and existence of neighboring nodes is the need to know sensor node's geographical location with respect to the sink node. This is obtained by some means like Global Positioning System (GPS) and localization techniques. In a prior work, we have proposed RSSI-based Forwarding (RBF) protocol that works without knowledge of node's location by using a Received Signal Strength Indicator (RSSI) level of beacon signals transmitted by the sink. Through contention, a next-hop node is determined among the forwarding candidates using a timer-based suppression scheme. We propose an improvement of the suppression scheme in which a contender closer to the sink is favored with a higher probability for being selected as a next-hop node. By means of simulation, it is shown that the performance of RBF is significantly improved using the enhanced mechanism.
\end{abstract}

Keywords: Cross-Layer Protocol; Medium Access Control; Routing; Wireless Sensor Networks.

\section{Introduction}

Wireless Sensor Networks (WSNs) are widely recognized as powerful means for in situ observations of events and environments over long periods of time [1. A sensor network consists of a large number of small sensor nodes with sensing, data processing, and communication capabilities which are densely deployed in a region of interest. Each node monitors its surrounding area and gathers application specific parameters (e.g., temperature, pressure, humidity, light, and chemical activity). The sensor nodes periodically sense data, process it and organize among themselves to form a communication network. They then collaborate to deliver the observations to a monitoring node, the sink node. The sensor nodes are typically powered with non-rechargeable batterries. Due to their limited transmission power, not all nodes can communicate directly with the sink 
node. Moreover, such communication would be over long distances that will drain power quickly. Multihop communication is then needed. The network must maintain the best connectivity as long as possible and it must be self-configured and self-organized in case of node failure. The possibility of node failure, wireless link failure and nodes transitioning into and out of sleep states to conserve energy introduce additional complexity to routing protocols that depend on up-to-date routing or neighborhood tables. This makes the routing state upkeep difficult. A routing solution that can deliver end-to-end traffic to a sink node without knowledge of network topology and the presence or absence of any other node is necessary, such a solution is defined as state-free in [2. It offers advantages in which a sender does not need to determine a forwarding node in advance or to find a path before the actual data transmission.

Several state-free routing protocols proposed in the literature 2/37/89] assume knowledge of nodes' geographical locations for routing the sensed data toward the sink node. With the WSN's characteristics that require a large number of low-cost and energy-efficient sensor nodes, equipping a GPS on every sensor node may not be practical. Furthermore, the cost of a GPS chip is much more expensive than the sensor node itself [14. Many location discovery protocols thus suggested to reduce or completely remove the dependence on GPS in WSNs 6101112 13.

In [5], we proposed the RBF protocol where routing and access are jointly managed, based on RSSI. In the preliminary work, RBF was tested on a topology with uniform deployment of sensor nodes. A simple propagation model was used where the path loss of a sensor node with respect to the sink was assumed as an increasing function of the distance. When nodes participate in the contention process, a suppression scheme to reduce the chance of selecting more than one node was made based on a uniform random choice of time slots within a contention window size. In this paper, we present an extension of such work by proposing an enhanced mechanism to select a next-hop node for the data forwarding task, taking into account the random effects of shadowing and testing of $\mathrm{RBF}$ in a random topology of sensor nodes.

The remainder of this paper is organized as follows. In section 2 , we give a detailed description of the protocol. Section 3 presents some simulation results and provides an analysis of the data collected. Finally, in section 4, we conclude this paper with a summary of our findings and future work.

\section{Protocol Description}

\subsection{Background and Assumptions}

The RBF protocol is described in [5]. For the sake of clarity, we hereby remind the reader of the main features of $\mathrm{RBF}$. We consider a wireless data collection network model with a large number of sensor nodes and one sink node. Each of the sensor nodes in the network is assumed to have a limited transmission power, and consequently limited transmission range. The data packets will then be relayed toward the sink using multihop communication. 
We assume the sink regularly transmits a beacon packet and the transmission power of the sink is high enough to reach all nodes in the network. Furthermore, the beacon packet includes the power level used for transmitting it. For an arriving beacon packet, each node measures the received power level of the beacon signal. To cope with radio strength instability, measurements may be made on several beacon packets. Each node then estimates an RSSI level that is an average over several samples. Node's mobility does not prevent the protocol from being functioning properly since new measurements of RSSI can always be refreshed based on regularly received beacon packets. By dividing the transmitted power of the beacon packet over the RSSI level, each node $i$ then knows the path loss $L_{i}$ between itself and the sink node.

\subsection{RBF Protocol Operation}

RBF protocol uses the four-way Request To Send (RTS)-Clear To Send (CTS)DATA-Acknowledgment (ACK) handshake as proposed in some schemes like XLM[4], IGF 2] and SIF [8]. Figure 1 shows the packet exchange sequence. The transmission starts with an RTS packet, which is broadcast. Nodes that receive an RTS packet check whether or not they are candidates to forward the data (see below). A CTS packet is then transmitted through a contention process. The node that wins the access is the forwarder. Finally, a normal DATA and ACK packet exchange follows between the two specific nodes. The process is repeated over multihop communications until the data is delivered to the sink.

We define a source node $i$ as the one that originates a data packet and a node $j$ which is within node $i$ transmission range as a neighbor node. When node $i$ has some data packet to transmit, it broadcasts an RTS packet to all its neighbor nodes. The RTS packet carries the path loss value of the sender (i.e. of $i$ ). Neighbor nodes that receive the RTS packet read the value and calculate the path loss ratio defined as their path loss divided by the path loss of $i$. If the

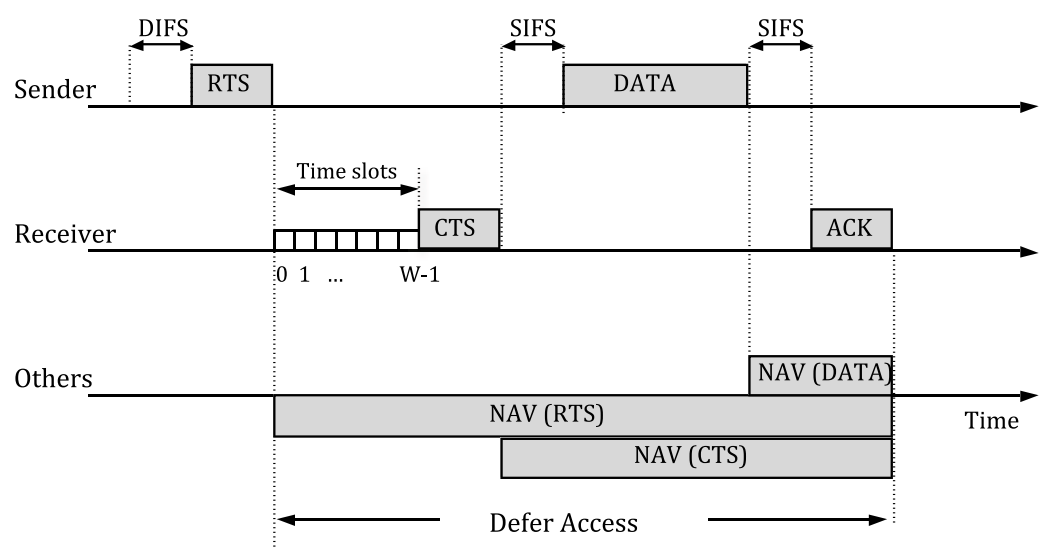

Fig. 1. Packet exchange sequence 
path loss ratio of the neighbor nodes to the source node is less than one, they participate in the contention process: a window of $W$ time slots is opened at the end of the RTS. Every candidate node randomly chooses a time slot. A node that chooses the lowest time slot wins the access. This node then responds with a CTS packet, establishing itself as the only next-hop node. Other nodes stop contending when they hear the CTS packet and adjust their network allocation vector (NAV), which indicate the amount of time that must elapse until the current transmission session is complete. Several nodes may decide to transmit a CTS packet at the same time. If there is a CTS packet collision, an RTS packet retransmission is performed.

\subsection{CTS Response Time}

In [5], we implemented a random backoff procedure for a node to respond with a CTS packet. A time slot is randomly chosen from a uniform distribution of time slots within a contention window size, $W$. Every forwarding node, therefore, has an equal probability of $\frac{1}{W}$ to select a time slot $k$ where $k \in[0, W-1]$. This promotes fairness among the forwarding nodes but may not be optimum since any neighbor node that has a path loss ratio less than one could send a CTS packet. We refer here the amount of time elapsed before replying to an RTS packet as CTS Response Time (CRT). For our discussion to follow, we refer to the random time slot selection scheme from a uniform distribution of time slots as Uniform CRT and an enhanced mechanism of CRT that we are currently proposing as Enhanced CRT.

The basic idea of Enhanced CRT is to make sensor nodes which are closer to the sink to take the forwarding responsibility with a higher probability. We propose the routing mechanism in RBF protocol as a function of path loss that gives an indication of whether a node is closer to the sink. However, due to the random effects of shadowing, this can not be assumed. For the same transmitter and receiver separation, different levels of clutter on the propagation path can be observed. A more distant node may then have a smaller path loss than a nearby one. Nevertheless, that gives a benefit to RBF protocol since data packet is always relayed toward the sink through a series of links with lesser path loss than the sending node. Since our metric of distance is based on path loss, this helps in the routing process to search for a higher quality link with lesser path loss to route the data packet toward the sink.

Let $L_{i}$ and $L_{j}$ be the path loss value between the sink and source node $i$, and node $j$, respectively. When node $j$ receives an RTS packet, $L_{i}$ is read from the packet. Knowing both $L_{j}$ and $L_{i}$, the path loss ratio $\frac{L_{j}}{L_{i}}$ is then used as a decision parameter for a node to participate in the contention process. We propose to choose a time slot $k$ where $k \in[0, W-1]$ with a probability $q p^{k}$ and with

$$
\begin{gathered}
p=b+\frac{1-b^{2}}{b}\left(\frac{L_{j}}{L_{i}}\right)^{\alpha}, \text { and } \\
q=\frac{1-p}{1-p^{W}} .
\end{gathered}
$$


Parameters $\alpha \in(0,1]$ and $b \in(0,1)$ in (11) are constants that are tunable to make a node closer to the sink to have a higher probability to send a CTS packet. It can easily be verified that $\sum_{k=0}^{W-1} q p^{k}=1$.

The impact of the path loss ratio $\frac{L_{j}}{L_{i}}, \alpha$ and $b$ parameters in (1) on the probability $q p^{k}$ is described as follows. If a neighbor node $j$ is closer to the sink with respect to the source node $i$, the path loss ratio $\frac{L_{j}}{L_{i}}$ will be close to zero. In this case, $p$ is approximately equal to $b$ and $q$ is approximately equal to $1-p$. The probabilities are higher near the time slot zero (see below), and therefore, we favor a forwarding node closer to the sink to respond first with a CTS packet. Other neighbor nodes with a higher path loss ratio tend to have a higher probability of choosing the last time slot of the contention window. Therefore, we can control and limit the probability of choosing the first and the last time slot.

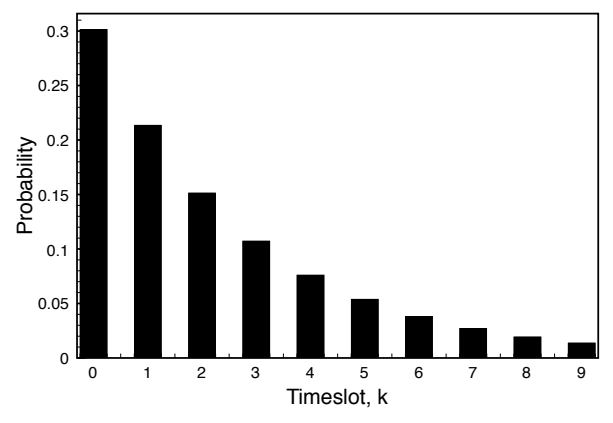

(a) $b=2 / 3, L_{j} / L_{i}=0.05$

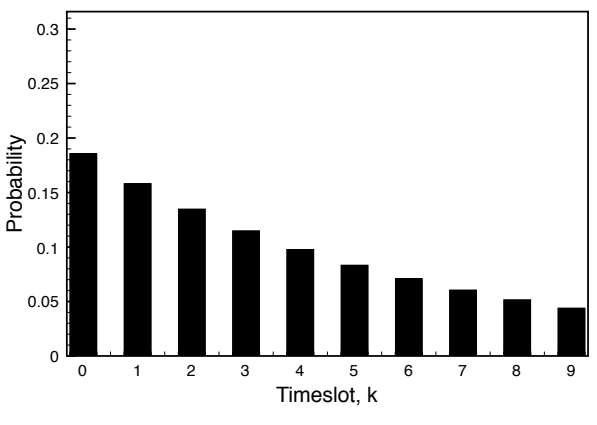

(b) $b=5 / 6, L_{j} / L_{i}=0.05$

Fig. 2. Probability distribution when node $j$ is closer to the sink than node $i$ among the forwarding nodes $\left(L_{j} / L_{i}=0.05\right)$. In (b), an increase in $b$, decreases the probability of time slot zero but increases the probability on the last time slot.

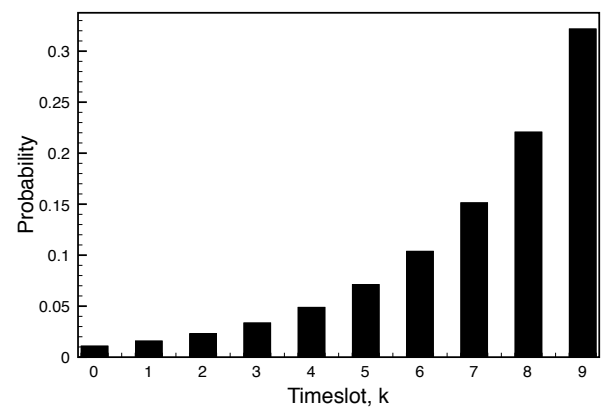

(a) $b=2 / 3, L_{j} / L_{i}=0.95$

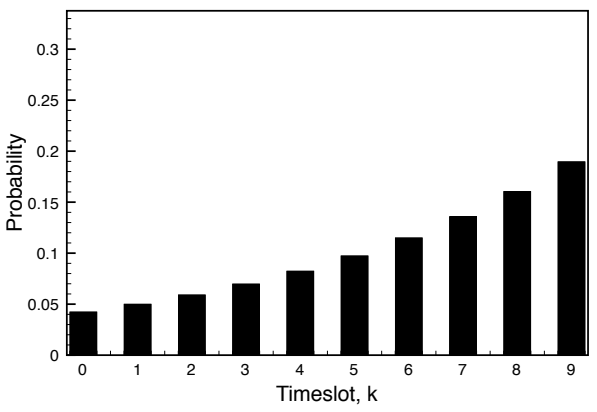

(b) $b=5 / 6, L_{j} / L_{i}=0.95$

Fig. 3. Probability distribution when node $j$ is closer to node $i$ among the forwarding nodes $\left(L_{j} / L_{i}=0.95\right)$. In (b), an increase in $b$, increases the probability of time slot zero but decreases the probability on the last time slot. 
By tuning appropriate values of $\alpha$ and $b$ in (1), we could favor a node $j$ closer to the sink to have a higher probability to respond with a CTS packet to node $i$. Varying the $b$ parameter affects the CRT of a forwarding node. When $b$ is increased, this disperses the probabilities of the time slots. For example, when $\alpha=1$ and $b=2 / 3$, the probability distribution of time slot selection from a contention window size, $W=10$ is shown in Fig. 2a when node $j$ is closer to the sink than node $i$, e.g., its path loss ratio $\frac{L_{j}}{L_{i}}=0.05$. With the same value of $\alpha$, an increase in $b$ decreases the probability of time slot zero but increases the probability on the last time slot. This is shown in Fig. 2b for $b=5 / 6$. However, when the path loss ratio is close to one, e.g., $\frac{L_{j}}{L_{i}}=0.95$, an increase in $b$ increases the probability of time slot zero but decreases the probability on the last time slot. The probability distributions for the path loss ratio $\frac{L_{j}}{L_{i}}=0.95$ are shown in Fig. 3a and Fig. 3b, for $b=2 / 3$ and $b=5 / 6$, respectively. Optimizing the values of $\alpha$ and $b$ is left for a future study.

\section{Performance and Simulation Results}

\subsection{Simulation Model}

To assess the performance of the RBF protocol, a simulation scenario was designed using OPNET@ Modeler [15]. A total of 112 sensor nodes are randomly deployed in an area of radius $105 \mathrm{~m}$. The sink is located in the center of the area. Table 1 shows some parameters used in the simulation. We assume the path loss of the signal varies according to the lognormal propagation model as in [16. Equation (3) gives the path loss $L$ at a distance $d$ from the transmitter node where $L_{0}$ is the path loss at a reference distance $d_{0}, \gamma$ is the path loss exponent, and $X_{\sigma}$ is a zero-mean Gaussian distributed random variable with a standard deviation $\sigma$.

$$
L=L_{0}+10 \gamma \log \left(\frac{d}{d_{0}}\right)+X_{\sigma} .
$$

\subsection{Simulation Results and Discussion}

We present simulation results where a number of sensor nodes that are most distant from the sink node generate data packets toward the sink in the center of the area. For such many-to-one communication, we evaluate the impact on the performance of RBF by varying the transmit power levels of the sensor nodes. Both simulation scenarios of Uniform CRT and Enhanced CRT mechanisms use five different transmit power levels: $3 \mathrm{~mW}(4.8 \mathrm{dBm}), 4 \mathrm{~mW}(6 \mathrm{dBm})$, $5 \mathrm{~mW}(7 \mathrm{dBm}), 6 \mathrm{~mW}(7.8 \mathrm{dBm})$ and $7 \mathrm{~mW}(8.5 \mathrm{dBm})$. For each transmit power level, the simulation was performed for 50 runs with 50 different random seeds.

Figure 4a shows the average number of hops for data delivery toward the sink for both Uniform CRT and Enhanced CRT mechanisms. Enhanced CRT improves the performance of RBF by favoring nodes closer to the sink to have 
Table 1. Simulation Parameters

\begin{tabular}{|c||c|}
\hline \multicolumn{1}{|c||}{ Parameter } & Value \\
\hline \hline Data rate & $250 \mathrm{kbits} / \mathrm{s}$ \\
\hline Slot time & $20 \mu \mathrm{s}$ \\
\hline SIFS time & $10 \mu \mathrm{s}$ \\
\hline Sensor node transmit power & 3 to $7 \mathrm{~mW}$ \\
\hline Sink transmit power (Beacon packet) & $1 \mathrm{~W}(30 \mathrm{dBm})$ \\
\hline Standard deviation of shadowing, $\sigma$ & $5 \mathrm{~dB}$ \\
\hline Data packet size & 32 Bytes \\
\hline Packet interarrival time for each node & Exponential $(60 \mathrm{~s})$ \\
\hline Contention window size,$W$ & 64 \\
\hline$\alpha$ & 1 \\
\hline$b$ & 0.833 \\
\hline & $300 \mathrm{~s}$ \\
\hline
\end{tabular}

a higher probability to send a CTS packet. This leads to a reduction of 22 to 27 percent in the average number of hops of the Uniform CRT for the same node transmit power level. As a result, the end-to-end (ETE) delay decreases. The ETE delay refers to the amount of time elapsed when a source node generates data packet and until it is delivered to the sink. Figure $4 \mathrm{~b}$ shows the distribution of hops for data delivery toward the sink for sensor node transmit power of $4 \mathrm{~mW}$. More hops are traversed by data packets using Uniform CRT since any forwarding contender with path loss ratio less than one may send a CTS packet with equal probability regardless of how far it is from the sink. With Uniform CRT, about 60 percent of data packets are routed through 6 to 10 hops to reach the sink and 40 percent are routed through 3 to 5 hops. On the other hand, with Enhanced CRT, about 89 percent of data packets are routed through 3 to 5 hops and 11 percent are routed through 6 to 7 hops. Consequently, the ETE delay is lower for the Enhanced CRT and higher for the Uniform CRT as depicted in Fig. 5a,

The ETE delay consists of propagation, transmission, processing and queuing delays. Propagation delay contributes a very small portion to the ETE delay, i.e., for a distance of $300 \mathrm{~m}$, the delay is only $1 \mu \mathrm{s}$. Transmission and processing delay is the time spent to emit bits onto the medium, receive, decode, and retransmit packet between source and destination. Queuing delay is the time spent by a packet waiting in a queue for transmission when the channel is busy, and depends on the congestion level of the wireless channel.

A variation of transmit power level affects the contention region at the MAC layer, in which it affects set of candidate nodes for next hop selection. A higher power level results in more nodes' participation in the contention process for sending a CTS packet. At the same time, an increase in transmit power increases the transmission range of nodes which then reduces the average number of hops each route needed in the network. Thus the total transmission delay along each route decreases. A lower power level results in shorter links, which means that 


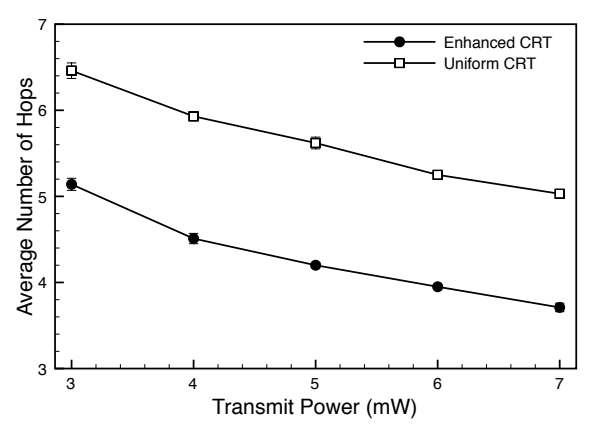

(a) Average number of Hops

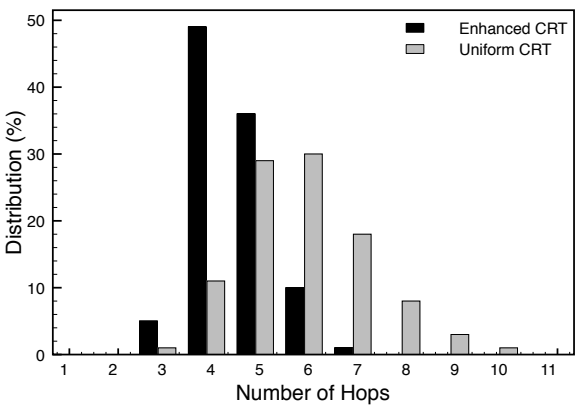

(b) Distribution of Hops

Fig. 4. Number of hops for data delivery toward the sink node. (a) average number of hops versus transmit power, and (b) distribution of hops for sensor node transmit power of $4 \mathrm{~mW}$.

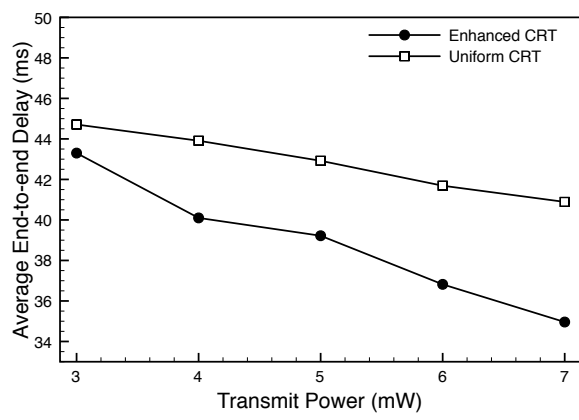

(a) Impact of transmit power on delay

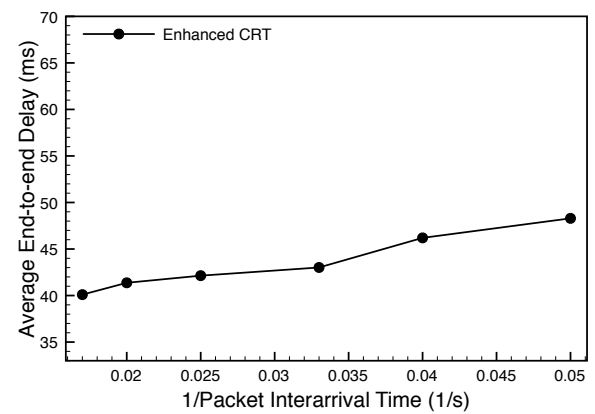

(b) Delay vs. packet interarrival time

Fig. 5. Average end-to-end delay for data delivery toward the sink node. (a) for node's packet interarrival time of $60 \mathrm{~s}$ with different transmit powers, and (b) for sensor node transmit power of $4 \mathrm{~mW}$ with different node's packet interarrival times.

more hops are required per route. This results in higher ETE delay and also puts more relaying burden on the nodes in the network. In this work, a lightly loaded network is considered, thus the queuing delay is small since number of nodes contending for channel access at the same time is small. In this case, transmission and processing delay is the dominant part of the ETE delay. For such loads, the packet delivery ratio is not degraded in the proposed Enhanced CRT mechanism. Uniform CRT promotes fairness among the forwarding nodes which means that all forwarding candidates have equal probability to respond with a CTS packet but results in higher ETE delay. On the other hand, Enhanced CRT favors nodes closer to the sink which results in lower ETE delay but perhaps it may put more relaying burden on some nodes in the network. How both mechanisms affect the 
overall nodes' relaying burden, energy consumptions and congestion level in the network deserves further investigation in a future study.

For a transmit power of $4 \mathrm{~mW}$, ETE delay with variation of nodes' packet interarrival times $(60,50,40,30,25$, and $20 \mathrm{~s})$ is also evaluated for Enhanced CRT as depicted in Fig. 5b With more data packets being generated by the sensor nodes, ETE delay increases.

\section{Conclusion}

In this paper, we have proposed an enhanced mechanism for a next hop node selection in the RBF protocol. A key component of the RBF protocol is node's path loss ratio which is used for the joint access/routing decision in the network. We have introduced probability functions in which forwarding candidates closer to the sink are favored with higher probability to take the responsibility for the data forwarding task. By varying node transmit power levels, its impact on the performance of the RBF is analyzed for both Enhanced CRT and Uniform CRT mechanisms. The RBF has been tested on a random topology of sensor nodes and with random effects of shadowing. Enhanced CRT has been shown to have an improved performance with lower average of hop counts and end-toend delay for data delivery toward the sink. As for the future work, we would like to explore the open issues highlighted in the paper and consider energy efficiency technique such that nodes' remaining energy levels are taken into account when forwarding candidates participate in the contention process, thus balancing energy consumptions in the network. A rigorous analysis of advantages and disadvantages of the power control in the cross-layer contention-based design of the multihop WSNs could be an interesting problem to explore too.

Acknowledgments. The work of A. Awang was partially supported by Universiti Teknologi PETRONAS, Malaysia.

\section{References}

1. Akyildiz, I.F., Su, W., Sankarasubramaniam, Y., Cayirci, E.: Wireless Sensor Networks: a survey. J. Computer Networks 38(4), 393-422 (2002)

2. Blum, B.M., He, T., Son, S., Stankovic, J.A.: IGF: A State-Free Robust Communication Protocol for Wireless Sensor Networks. In: Technical Report CS-2003-11, CS Department, University of Virginia (2003)

3. Füßler, H., Widmer, J., Käsemann, M., Mauve, M., Hartenstein, H.: Contentionbased forwarding for mobile ad hoc networks. J. Ad Hoc Networks 1(4) (2003)

4. Akyildiz, I.F., Vuran, M.C., Akan, O.B.: A Cross-Layer Protocol for Wireless Sensor Networks. In: Proc. Conference on Information Science and Systems (CISS 2006), Princeton, NJ, March 22-24 (2006)

5. Awang, A., Lagrange, X., Ros, D.: A Cross-Layer Medium Access Control and Routing Protocol for Wireless Sensor Networks. In: Proc. 10èmes Journées Doctorales en Informatique et Réseaux (JDIR 2009), February 2-4 (2009) 
6. Liu, D., Ning, P., Du, W.: Detecting Malicious Beacon Nodes for Secure Location Discovery in Wireless Sensor Networks. In: Proc. IEEE ICDCS (2005)

7. Chen, D., Deng, J., Varshney, P.K.: On the Forwarding Area of Contention-Based Geographic Forwarding for Ad Hoc and Sensor Networks. In: Proc. IEEE SECON (2005)

8. Chen, D., Deng, J., Varshney, P.K.: A State-Free Data Delivery Protocol for Multihop Wireless Sensor Networks. In: Proc. IEEE WCNC (2005)

9. Zorzi, M., Rao, R.R.: Geographic Random Forwarding (GeRaF) for Ad Hoc and Sensor Networks: Multihop Performance. J. IEEE Trans. on Mobile Computing 2(4) (October-December 2003)

10. Bulusu, N., Heidemann, J., Estrin, D.: GPS-less Low-Cost Outdoor Localization for Very Small Devices. In: IEEE Personal Commun. Mag., pp. 28-34 (2000)

11. He, T., Huang, C., Blum, B.M., Stankovic, J.A., Abdelzaher, T.: Range-Free Localization Schemes for Large Scale Sensor Networks. In: Proc. ACM MOBICOM (2003)

12. Nasipuri, A., Li, K.: A Directionality based Location Discovery Scheme for Wireless Sensor Networks. In: Proc. ACM WSNA (2002)

13. Savvides, A., Han, C., Strivastava, M.B.: Dynamic Fine-Grained Localization in Ad-Hoc Networks of Sensors. In: Proc. ACM MOBICOM (2001)

14. Heo, J.H., Kim, J.-H., Hong, C.S.: A logical group formation and management mechanism using RSSI for wireless sensor networks. In: May, Y., Choi, D., Ata, S. (eds.) APNOMS 2008. LNCS, vol. 5297, pp. 207-216. Springer, Heidelberg (2008)

15. OPNET Technologies, Inc., http://www.opnet.com/

16. Rappaport, T.S.: Wireless Communications: Principles and Practice. Prentice Hall, New Jersey (2001) 